\title{
Evaluation of skin sensitization based on interleukin-2 promoter activation in Jurkat cells
}

\author{
TAICHI NAGAHATA ${ }^{1}$, YOSHIO TSUJINO $^{2}$, EIJI TAKAYAMA $^{3}$, HARUKA HIKASA $^{1}$ and AYANO SATOH ${ }^{1}$ \\ ${ }^{1}$ Graduate School of Interdisciplinary Science and Engineering in Health Systems, Okayama University, Okayama 700-0082; \\ ${ }^{2}$ Graduate School of Science, Technology and Innovation, Kobe University, Kobe, Hyogo 657-8501; \\ ${ }^{3}$ Department of Oral Biochemistry, Asahi University School of Dentistry, Gifu 501-0296, Japan
}

Received August 19, 2021; Accepted October 8, 2021

DOI: $10.3892 / \mathrm{br} .2021 .1486$

\begin{abstract}
Skin sensitization is an allergic reaction caused by certain chemical substances, and is an important factor to be taken into consideration when evaluating the safety of numerous types of products. Although animal testing has long been used to evaluate skin sensitization, the recent trend to regulate such testing has led to the development and use of alternative methods. Skin sensitization reactions are summarized in the form of an adverse outcome pathway consisting of four key events (KE), including covalent binding to skin proteins (KE1), keratinocyte activation (KE2), and dendritic cell activation (KE3). Equivalent alternative methods have been developed for KE1 to KE3, but no valid alternative has yet been developed for the evaluation of KE4 and T-cell activation. Current alternative methods rely on data from KE1 to KE3 to predict the effect of chemicals on skin sensitization. The addition of KE4 data is expected to improve the accuracy and reproducibility of such predictions. The aim of this study was to establish an assay to evaluate KE4 T-cell activation to supplement data on skin sensitization related to KE4. To evaluate T-cell activation, the Jurkat T-cell line stably expressing luciferase downstream of the pro-inflammatory cytokine interleukin-2 promoter was used. After exposure to known skin sensitizing agents and control substances, luciferase activity measurements revealed that this assay was valid for evaluating skin sensitization. However, two skin sensitizers known to have immunosuppressive effects on T-cells reacted negatively in this assay. The results revealed that this assay simultaneously allows for monitoring of the skin sensitization and immuno-suppressiveness of chemical substances and supplements KE4 T-cell activation data, and may thus contribute to reducing the use of animal experiments.
\end{abstract}

Correspondence to: Dr Ayano Satoh, Graduate School of Interdisciplinary Science and Engineering in Health Systems, Okayama University, Tsushima-naka 3-1-1, Kita-ku, Okayama 700-0082, Japan E-mail: ayano113@cc.okayama-u.ac.jp

Key words: skin sensitization, immunotoxicity, interleukin-2 promoter, Jurkat, T-cell activation

\section{Introduction}

Allergic dermatitis caused by skin sensitizers is one of the most important factors that should be taken into consideration when evaluating the safety of chemical substances. Skin sensitization is classified as type IV hypersensitivity (delayed reaction), involving immune cells, such as T-cells (1). In allergic dermatitis caused by skin sensitization, inflammatory reactions peak 24 to $48 \mathrm{~h}$ after contact with the causative agent. Allergic contact dermatitis accounts for $\sim 60 \%$ of all occupational skin diseases. Although skin sensitization is not a life-threatening disease, it greatly affects social life and can result in issues with regard to careers, particularly in patients dealing with consumer products, reviewed in (2).

An allergy consists of two aspects: The sensitization stage, in which a person acquires reactivity to a substance, and the elicitation stage, in which allergic symptoms develop. In skin sensitization, when a chemical substance is repeatedly absorbed transdermally, T-cells that specifically react to a chemical substance proliferate in the body, and sensitization is established. When the same chemical is transdermally absorbed again, the reacting $\mathrm{T}$-cells release cytokines and induce inflammation at the contact site (triggering). Knowledge of the chemical and biological mechanisms involved in skin sensitization reactions is summarized in the adverse outcome pathway (AOP) that includes initial events at the molecular level through intermediate events to adverse effects (3). The initial event at the molecular level, or the first key event (KE1), is the covalent bonding of the nucleophilic center of a protein with an electrophilic substance present in the skin. KE2 is the covalent bonding of the electrophilic substance with a specific cell signaling pathway, such as the inflammatory response in keratinocytes or the antioxidant/electrophile response sequence-dependent pathway. KE3 is the activation of dendritic cells (DCs), and KE4 is the activation of T-cells. The skin sensitization assay is an in vivo or in vitro replication of all or part of the AOP. Animal experiments, such as the guinea pig maximization test (4) and mouse local lymph node assay (5), have been used to evaluate skin sensitization.

Recent stricter regulations on animal experiments aim to ensure animal welfare by following the basic principle of animal testing defined by the 3Rs: Reduction, refinement and replacement; replacement that signifies the complete elimination of 
animal testing is the most important issue, reviewed in (6). In the EU, the leader in regulating animal testing in the cosmetics industry, animal testing for cosmetics started to be gradually banned in 2004. Since March 2013, there has been a complete ban on the sale and import of all raw materials, processed products containing raw materials, and finished products tested on animals (7), and manufacturers exporting these raw materials to the EU have been forced to respond. Not only for the cosmetics industry, but also for other industries, including pharmaceutical tests that likely accounts for the majority of animal testing, reducing animal experiments would be preferred in terms of animal welfare. Therefore, the development and use of alternative methods for animal testing, such as in vitro and in chemico testing are gaining importance. Currently, the OECD Test Guidelines list the following alternative methods for skin sensitization tests: The direct peptide reactivity assay (DPRA), amino acid derivative reactivity assay (ADRA) for KE1 (8), KeratinoSens ${ }^{\mathrm{TM}}$ for KE2 (9), the human cell line activation test (h-CLAT), U-SENS ${ }^{\mathrm{TM}}$ and IL-8 Luc assay for KE3 (10). However, an effective alternative method for KE4 'T-cell activation' has yet to be fully developed, representing a challenge in this field.

KE4 in skin sensitization AOP involves activation of naïve T-cells, recognition of antigen presentation by DCs, and proliferation of antigen-specific effector T-cells and memory T-cells; it plays an important role in the overall AOP (11). These responses occur through complex biological processes. The activation of naïve T-cells requires two signals that act synergistically. The first major signal results from the binding of major histocompatibility complex (MHC)-antigen peptide complexes presented by antigen-presenting cells (APCs), such as DCs, to T-cell receptors (TCRs) on T-cells. The second major signal is derived from the interaction of costimulatory receptors on T-cells with ligands on APCs. A typical costimulatory receptor is $\mathrm{CD} 28$ that interacts with the corresponding ligands on APCs, CD80 and CD86. In addition to the two major signals, cytokines are essential for T-cell activation. For example, interleukins such as IL- $1 \alpha$, IL-1 $\beta$, IL-18 and TNF- $\alpha$ are required for the migration of APCs from the skin to lymph nodes and the presentation of MHC-antigen peptide complexes, reviewed in (12). Owing to the complexity of these processes, it is difficult to fully reproduce these events in vitro.

In the present study, an evaluation system for some of these complex processes was established and proposed. interleukin-2 (IL-2) expression in T-cells was used as an indicator to evaluate the KE4 of skin-sensitizing AOPs. IL-2, a pro-inflammatory cytokine, is released from activated T-cells and plays an important role in the immune response by activating immune-related cells, such as memory T-cells and natural killer (NK) cells (13). Therefore, it was hypothesized that the evaluation of IL-2 activity in T-cells could provide important information for evaluating the KE4. The Jurkat clone E6-1 human T-lymphocyte immortalized cell line is frequently used in studies of human T-cell line activation in vitro (14). In the present study, Jurkat cells (IL-2p::Jurkat cells) that express the luciferase gene downstream of the IL-2 promoter (IL-2p) were used. Using five skin sensitizers, including a typical skin sensitizer 2,4-dinitrochlorobenzene (DNCB), and two non-sensitizers as test substances, the activation of the IL-2 promoter as a marker of T-cell activation was evaluated to determine the validity of this assay.

\section{Materials and methods}

Cell culture. IL-2p::Jurkat cells (cat. no. J1651; Promega Corporation; within 10-20 passages after obtaining the cell line) (15) were maintained in RPMI-1640 medium supplemented with $10 \% \mathrm{FBS}$ at $37^{\circ} \mathrm{C}$ and $5 \% \mathrm{CO}_{2}$. The cell suspension was mixed with an equal volume of Trypan blue solution (Nacalai Tesque Inc.), and the number of viable cells, number of dead cells and cell viability were recorded using a hemocytometer. Once every 2-3 days, the cells were seeded at a density of $1-2 \times 10^{5}$ cells $/ \mathrm{ml}$, and a density of $0.1-1.0 \times 10^{6}$ cell $/ \mathrm{s} / \mathrm{ml}$ was maintained.

Determination of the chemical concentration for $90 \%$ cell viability (CV90). All chemicals used in the present study were obtained from Sigma-Aldrich (Merck KGaA) or Tokyo Chemical Industry. The concentrations of chemicals used for cell viability studies were determined by referring to the exposure experiments on Jurkat cells $(11,16)$. Saline was used as a solvent for $\mathrm{NiSO}_{4}$, glyoxal and lactic acid, and DMSO was used as a solvent for the other chemicals.

IL-2p::Jurkat cells were transferred from flasks to $15 \mathrm{ml}$ tubes, centrifuged $\left(250 \mathrm{x} \mathrm{g}, 5 \mathrm{~min}, 25^{\circ} \mathrm{C}\right)$, and the supernatant was removed. The cell pellet was resuspended in fresh medium to a concentration of $1 \times 10^{6}$ cells $/ \mathrm{ml}$. A total of $60 \mu \mathrm{l}$ of the resuspended cells were added to a 96-well plate (flat-bottom, transparent), and $30 \mu \mathrm{l}$ PBS or test chemicals shown in Table I were added to each well containing the IL-2p::Jurkat cell suspension. WST-1 assay reagent ( $3 \mu \mathrm{l}$; cat. no. MK400; Takara Bio, Inc.) was added and cells were incubated for $1 \mathrm{~h}$ at $37^{\circ} \mathrm{C}$ with $5 \% \mathrm{CO}_{2}$. After incubation, the absorbance at $450 \mathrm{~nm}$ (control wavelength $670 \mathrm{~nm}$ ) was measured using an iMark ${ }^{\mathrm{TM}}$ microplate reader (Bio-Rad Laboratories, Inc.), and relative cell viability and CV90 were determined using Equations 1 and 2, respectively; Equation 1: Relative cell viability $=\left[\left(\mathrm{A}_{450}\right.\right.$ of cells exposed to chemical)-( $\mathrm{A}_{670}$ of cells exposed to chemical $)] /\left[\left(\mathrm{A}_{450}\right.\right.$ of cells exposed to PBS $)-\left(\mathrm{A}_{670}\right.$ of cells exposed to PBS $)]$, and Equation 2: $\log C V 90=[(90-c) \times \log (b)-(90-a)$ $\mathrm{x} \log (\mathrm{d})] /(\mathrm{a}-\mathrm{c})$, where $\mathrm{a}$ is the minimum viability at which the cell viability is $>90 \%$, $\mathrm{c}$ is the maximum viability at which the cell viability was $<90 \%$, and $b$ and $d$ are the concentrations at the cell viability of a and c, respectively.

Exposure of IL-2p::Jurkat cells to chemicals and their evaluation. For the pre-activation of T-cells, anti-CD3 pre-coated 96-well plates were prepared. A total of $6 \mu 1$ of a stock solution of anti-CD3 antibody (Clone OKT-3; cat. no. ab86883; Abcam) was diluted in 1,200 $\mu 1$ PBS. The diluted anti-CD3 antibody solution was added to a 96 -well plate (60 ng/well) and incubated at $4^{\circ} \mathrm{C}$ overnight. After incubation, the solution was removed with an aspirator, and $100 \mu \mathrm{l}$ culture medium (RPMI-1640 + 10\% FBS) was added to each well. This process was repeated twice (insufficient washing affects cell growth). A total of $30 \mu \mathrm{l}$ IL-2p::Jurkat cell suspension $\left(1 \times 10^{6}\right.$ cells $\left./ \mathrm{ml}\right)$ was then added to each 96-well plate, and $15 \mu \mathrm{l}$ PBS was added to the negative control, or anti-CD28 antibody solution as a positive control (2 $\mu \mathrm{g} / \mathrm{ml}$; clone CD28.2; cat. no. 12-577-C100; Exbio-Funakoshi, Co., Ltd.) and the chemical solutions were added. The cells were then incubated for $9 \mathrm{~h}$ at $37^{\circ} \mathrm{C}$, and luminescence was measured using a GloMax ${ }^{\circledR}$ Navigator 
Table I. CV90 of the chemicals used in this study.

\begin{tabular}{|c|c|c|c|c|c|}
\hline Chemical & LLNA $^{\mathrm{a}}$ & h-CLAT ${ }^{a}$ & DPRA $^{a}$ & $\mathrm{CV} 90^{\mathrm{c}}$ & Solvent used \\
\hline DNCB & Strong & + & + & 1.059 & DMSO \\
\hline $\mathrm{NiSO}_{4}$ & Moderate & + & + & 27.13 & Saline \\
\hline Isoeugenol & Moderate & $-{ }^{b}$ & + & 84.15 & DMSO \\
\hline Diethylenetriamine & Moderate & $-{ }^{b}$ & + & 716.7 & DMSO \\
\hline Glyoxal & Moderate & + & + & 30.20 & Saline \\
\hline Benzyl benzoate & Weak & $-{ }^{b}$ & - & 276.0 & DMSO \\
\hline Lactic acid & Negative & - & - & 1203 & Saline \\
\hline
\end{tabular}

${ }^{\text {aS }}$ kin sensitization test results from (12). ${ }^{\mathrm{b}}$ Chemicals exhibiting responses positive to LLNA but negative to h-CLAT. ${ }^{\mathrm{c}} \mathrm{CV} 90$ was determined using Equations 1 and 2 (see the Materials and methods section) and the data used for the determination are shown in Tables SI. LLNA, local lymph node assay; h-CLAT, human cell line activation test; DPRA, direct peptide reactivity assay; CV90, chemical concentration for $90 \%$ cell viability; DNCB, 2,4-dinitrochlorobenzene.

Microplate Luminometer (Promega Corporation) after adding $7 \mu$ l Bio-Glo reagent (cat. no. G7941; Promega Corporation) to each well according to the manufacturer's instructions. IL-2p (IL-2 promoter) induction was calculated using Equation 3; Equation 3: IL-2p induction $=$ [(luminescence of cells exposed to test chemicals]-[luminescence of cells exposed to PBS)] $\mathrm{x} 100 /[($ luminescence of cells exposed to anti-CD28)-(luminescence of cells exposed to PBS)].

\section{Results}

Determination of CV90. Reasonable alternative assays for the animal experiments used for evaluation of KE4 of skin sensitizing AOP are currently not available. In the present study, the proliferation of an immortalized T-cell line, Jurkat cells, that stably express luciferase under downstream of the IL-2 promoter (IL-2p::Jurkat) were used to assist in method development. The skin sensitizers and control chemicals used in this study are listed in Table I. Chemicals marked with 'b' exhibit local lymph node assay-positive but h-CLAT-negative response. The skin sensitivities were also verified using the assay developed in this study, which was termed the IL-2p::Jurkat assay.

To determine the appropriate concentration of the chemicals, cells were exposed to various concentrations of the compounds, and CV90 was determined using Equations 1 and 2, as shown in Table I.

Evaluation of skin sensitization by IL-2p::Jurkat assay. IL-2p::Jurkat cells were pre-activated by plating into anti-CD3 pre-coated 96-well plates. Test chemicals were then added, incubated for $9 \mathrm{~h}$, and luciferase activity corresponding to IL-2 promoter activation was measured to determine cell proliferation. IL-2p induction was determined using Equation 3.

Exposure to the skin sensitizers DNCB, diethylenetriamine and glyoxal increased IL-2 promoter activity, whereas exposure to the weak or non-sensitizing substances benzyl benzoate and lactic acid did not. That is, the IL-2p::Jurkat assay correctly reflected the skin sensitivity to these five subst ances (Fig. 1 and Table II). However, this assay did not detect skin sensitivity to $\mathrm{NiSO}_{4}$ and isoeugenol and showed inhibition of T-cell proliferation.
Possibility of simultaneous evaluation of skin sensitization and immunosuppressiveness. There are two types of immunotoxicity: Immunosuppressive and hyper-immunogenic $(17,18)$. Initially, the aim was to develop an assay to evaluate skin sensitization, a feature of hyper-immunotoxicity. However, as mentioned above, $\mathrm{NiSO}_{4}$ and isoeugenol, which exert an immunosuppressive effect on T-cells, suppressed the expression of IL-2. Therefore, in the present study, it may be possible to simultaneously evaluate immunotoxicity in terms of both immunosuppressive and hyper-immunogenic properties based on the degree of suppression and enhancement of IL-2 expression. The maximum and minimum values of IL- $2 p$ induction for each chemical in this study are listed in Table II.

As shown in Table II, the weak or non-sensitizing substances benzyl benzoate and lactic acid had maximum and minimum values of IL- $2 p$ induction within \pm 20 . Therefore, the test developed in the present study may be used as a simple screening test for immunotoxicity of chemical substances by defining 'IL-2p induction $\geq 20$ ' as hyper-immunogenic/immunotoxic (a skin sensitizer), 20> IL-2p induction $>-20$ or as negatively immunotoxic, and 'IL-2p induction $\leq-20$ ' as immunosuppressive/immunotoxic (Table III).

\section{Discussion}

In the present study, an IL-2p::Jurkat assay for determining the skin sensitization AOP KE4 T-cell activation was designed and evaluated. The inflammatory cytokine IL-2 is a typical cytokine produced by activated T-cells; this assay has been shown to correctly evaluate the activation of T-cells by chemicals. However, it is unclear at which stage of the skin sensitization response Jurkat cells reflect T-cell activation in vivo. The two major time points when T-cells are activated in the skin sensitization response are as follows: First, when naïve T-cells initially recognize antigens presented by DCs, and second, when antigen-specific memory T-cells are already sensitized and proliferating and recognize antigens presented by DCs. Normally, it takes repeated exposure to a chemical and some time for naïve T-cells to recognize it and for sensitized T-cells to proliferate (19). In the test system used in this study, the time of exposure of the cells to the chemical substance was relatively 
Table II. Maximum/minimum interleukin-2 promoter induction at the indicated CV90.

\begin{tabular}{lccc}
\hline Chemical & Maximum $^{\mathrm{a}}(\mathrm{CV} 90$ fraction $)$ & Minimum $^{\mathrm{a}}(\mathrm{CV} 90$ fraction) & Prediction using Table III \\
\hline $\mathrm{DNCB}$ & $113.9(\mathrm{CV} 90)$ & $12.5(\mathrm{CV} 90 / 3)$ & Hyper-immunogenic \\
$\mathrm{NiSO}_{4}$ & $-4.9(\mathrm{CV} 90 / 3)$ & $-27.9(\mathrm{CV} 90)$ & Immunosuppressive \\
Isoeugenol & $-7.0(\mathrm{CV} 90 / 3)$ & $-26.3(\mathrm{CV} 90)$ & Immunosuppressive \\
Diethylenetriamine & $39.9(\mathrm{CV} 90 / 1.5)$ & $13.3(\mathrm{CV} 90 / 3)$ & Hyper-immunogenic \\
Glyoxal & $187.2(\mathrm{CV} 90)$ & $60.3(\mathrm{CV} 90 / 3)$ & Hyper-immunogenic \\
Benzyl benzoate & $4.9(\mathrm{CV} 90)$ & $-7.8(\mathrm{CV} 90 / 1.5)$ & Negative \\
Lactic acid & $4.7(\mathrm{CV} 90 / 3)$ & $-13.2(\mathrm{CV} 90)$ & Negative \\
\hline
\end{tabular}

aOriginal data to determine these values are shown in Fig. 1. CV90/1.5, CV90/2 or CV90/3 mean CV90 divided by 1.5, 2 or 3, respectively. CV90, chemical concentration for $90 \%$ cell viability; DNCB, 2,4-dinitrochlorobenzene.

Table III Proposed new index for T cell activation.

\begin{tabular}{ll}
\hline Interleukin-2 promoter induction & \multicolumn{1}{c}{ Prediction } \\
\hline Induction $\geq 20$ & $\begin{array}{l}\text { Hyper-immunogenic } \\
\text { (skin sensitizer) }\end{array}$ \\
Inductionl $<20$ & Negative \\
Induction $\leq-20$ & Immunosuppressive \\
\hline
\end{tabular}

short $(9 \mathrm{~h})$ and required pre-activation by anti-CD3, so the activation of antigen-specific memory T-cells that have already been sensitized was likely being evaluated rather than naïve T-cells.

Three possible reasons for the false-negative result in which the assay did not detect skin sensitivity to $\mathrm{NiSO}_{4}$ and isoeugenol, and showed inhibition of T-cell proliferation, are as follows: i) The immunosuppressive effect on T-cells: Nickel and isoeugenol have been reported to exert immunosuppressive effects on T-cells and suppress IL-2 expression (20,21). Note that the word 'immunosuppression' is used here according to the previous literature $(20,21)$. Whether the expression of other cytokines and cell surface antigens were altered by these agents to determine their suppression or regulation was not performed in the present study. ii) The lack of DCs: Nickel has been reported to exert an immunosuppressive immunotoxic effect on T-cells but induces cell maturation via NF- $\kappa \mathrm{B}$ and MAPK signaling pathways in antigen-presenting DCs (20). DCs were absent in the in vitro test; nickel and isoeugenol, having an immunosuppressive effect on T-cells alone, may exert hyperimmunogenicity when interacting with DCs; hence, the absence of DCs may have caused this false-negative result. iii) Pre/pro-hapten issue; isoeugenol is a pre/pro-hapten chemical that is prone to air oxidation and metabolism $(22,23)$; a pre/pro-hapten such as isoeugenol could be a false negative in the test in the absence of a metabolic system.

The IL-2 expression of T-cells evaluated in this study is a part of the skin sensitization AOP KE4 'T-cell activation'. To complement KE4, other indicators, such as the expression of IFN- $\gamma$ and other molecules, the proliferation of antigen-specific memory T-cells, expression of granzyme B in CD8 ${ }^{+} \mathrm{T}-$-cells, and other assay systems, such as adopting stimulation from DCs, oxidation and metabolism need to be considered.
The concentration of chemicals tested with IL-2p::Jurkat cells in the present study were determined based on the CV90 value; this correctly determined the skin sensitization of the five chemicals better than CV75 used in KeratinoSens and hCLAT (12); therefore, the use of CV90 is considered appropriate. However, if false-negative results are obtained when testing weaker skin sensitizers in the future, it will be necessary to re-examine the effect of exposure concentrations. It is important to note that exposure to high concentrations of highly cytotoxic chemicals as well as skin sensitizers, such as DNCB, may cause cell death, producing unreliable results; the duration of chemical exposure may be another factor to be re-examined in the case of false-negative results when testing weaker skin sensitizers.

In the future, the accuracy and validity of the test will be assessed by evaluating more chemicals using IL-2p::Jurkat cells. Agents that are unstable in the atmosphere or water were not assessed to avoid inaccuracies in the evaluations, although it would be better to have a greater number of test chemicals evaluated for the further validation. In addition, evaluation indices other than IL-2 mentioned above will be used to complement the skin sensitization evaluation data related to KE4 by combining the test system with the test system using CD69 as an index, as this has already been examined (11), and/or the THP-1 cell and CD4+ T-lymphocyte co-culture system $(24,25)$.

As described in the Materials and methods, FBS for Jurkat cell culture was used, although it was minimal. FBS is widely used for cell culture; therefore, there is a large amount of accumulated data. Thus, it was decided that it would be beneficial and necessary to compare and confirm our data obtained using past accumulated data. Since FBS is derived from animals, there is a potential concern that its performance depends on its batch $(26,27)$. Furthermore, there have been ethical concerns regarding FBS and discussions about a fully chemically defined medium to replace FBS $(26,27)$. Although limited, FBS alternatives have been developed and used for particular purposes, for example, non-allergenic purposes. Therefore, FBS alternatives for Jurkat cell culture should be considered in the future. Additionally, as described in the Materials and methods, anti-CD3 and anti-CD28 mouse monoclonal antibodies obtained from pharmaceutical companies were used. Although information on their origins is unavailable, they were likely purified from ascites. These antibodies should be replaced with those that can be confirmed to have been purified from hybridomas expressing anti-CD3 (OKT-3) and anti-CD28 (28.2), which would 

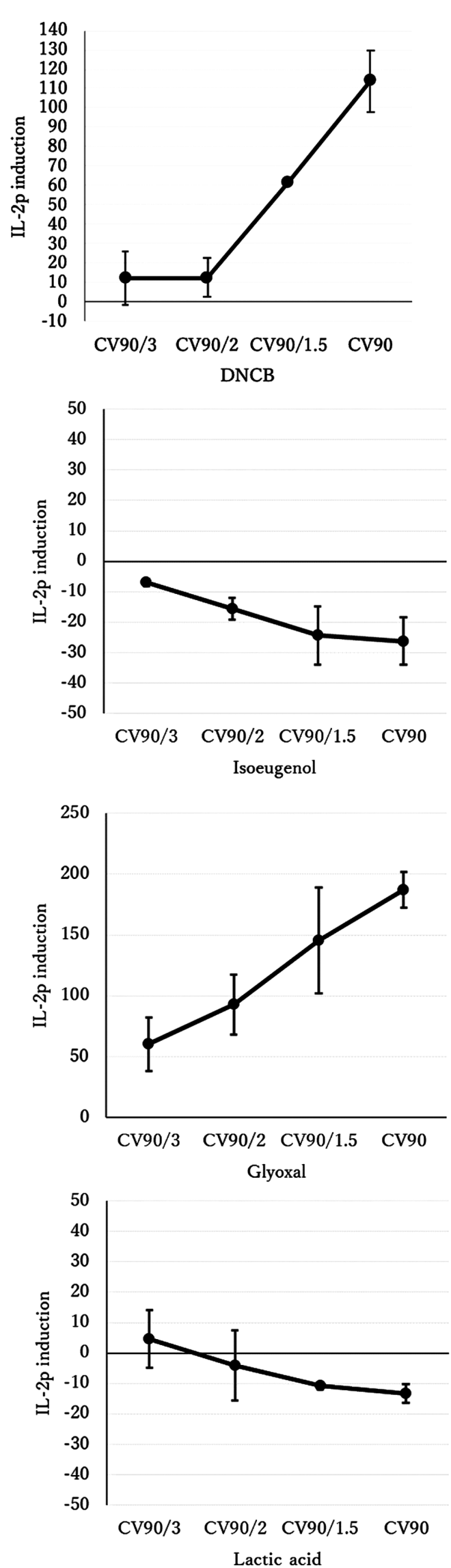
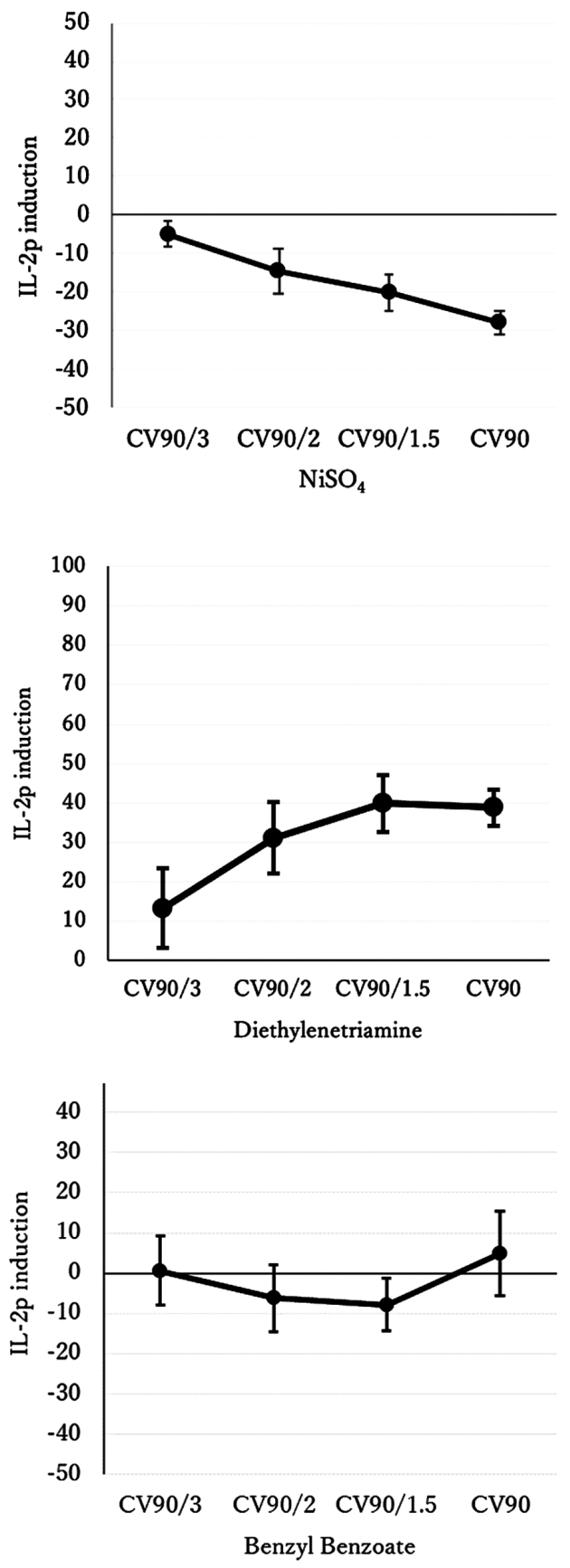

Figure 1. Relative IL-2p induction. IL-2p::Jurkat cells were exposed to four concentrations (CV90, CV90/1.5, CV90/2, CV90/3) of the indicated chemicals, incubated for $9 \mathrm{~h}$, and then IL-2p induction was measured. The vertical axis is the IL-2p induction where cell exposure to PBS was set to 0 as a negative and background control, and cell exposure to anti-CD28 was set to 100 as a positive control. The values in the graph represent the mean \pm standard deviation of three independent experiments. The concentrations and CVs that indicate maximum and minimum induction are shown in Table II. Importantly, CV90/1.5, CV90/2, or CV90/3 refer to CV90 divided by $1.5,2$ or 3, respectively. CV, cell viability; IL-2p, interleukin-2 promoter. 
further reduce the use of animal-derived materials. Ideally, these antibodies should be replaced with identical recombinant antibodies purified using animal-free materials if such antibodies are available in the future.

In conclusion, the alternative method to evaluate KE4 of skin sensitization proposed in this study, if further developed, may eliminate the need for conventional skin sensitization experiments that cause suffering and pain in animals, thus contributing to the reduction of animal testing.

\section{Acknowledgements}

The authors would like to thank Dr Mayuko Koreishi (Okayama University, Okayama, Japan) for cell culture and technical assistance.

\section{Funding}

This work was supported in part by MEXT/JSPS KAKENHI (grant no. 18K06133) and the Okayama Prefecture Tokubetsu Dengen.

\section{Availability of data and materials}

All data generated and/or analyzed during this study are included in this published article.

\section{Authors' contributions}

TN and AS performed the experiments and analyzed the data. TN, YT, ET, HH and AS contributed to the conception and design of the study. TN and AS contributed to data acquisition and analysis. All authors read and approved the final manuscript. YT, ET and HH confirm the authenticity of all the raw data.

\section{Ethics approval and consent to participate}

Not applicable.

\section{Patient consent for publication}

Not applicable.

\section{Competing interests}

The authors declare that they have no competing interests.

\section{References}

1. van Vliet E, Kühnl J, Goebel C, Martinozzi-Teissier S, Alépée N, Ashikaga T, Blömeke B, Del Bufalo A, Cluzel M, Corsini E, et al: State-of-the-art and new options to assess T cell activation by skin sensitizers. ALTEX 35: 179-192, 2018.

2. Qin R and Lampel HP: Review of occupational contact dermatitis - Top allergens, best avoidance measures. Curr Treat Options Allergy 2: 349-364, 2015.

3. Organisation for Economic Co-operation and Development: The Adverse Outcome Pathway for Skin Sensitisation Initiated by Covalent Binding to Proteins. Available from: https://doi org/10.1787/9789264221444-en.

4. Organisation for Economic Co-operation and Development: Test No. 406: Skin Sensitisation. 1992. Available from: https://doi org/10.1787/9789264070660-en.

5. Organisation for Economic Co-operation and Development: Test No. 429: Skin Sensitisation. 2010. Available from: https://doi. org/10.1787/9789264071100-en.
6. Herrmann K, Pistollato F and Stephens ML: Beyond the 3Rs: Expanding the use of human-relevant replacement methods in biomedical research. ALTEX 36: 343-352, 2019.

7. European Commision: Ban on Animal Testing: Internal Market, Industry, Entrepreneurship and SMEs. Available from: https:// ec.europa.eu/growth/sectors/cosmetics/animal-testing_en.

8. Organisation for Economic Co-operation and Development: Test No. 442C: In Chemico Skin Sensitisation. 2020. Available from: https://doi.org/10.1787/9789264229709-en.

9. Organisation for Economic Co-operation and Development: Test No. 442D: In Vitro Skin Sensitisation. 2018. Available from: https://doi.org/10.1787/9789264229822-en.

10. Organisation for Economic Co-operation and Development: Test No. 442E: In Vitro Skin Sensitisation. 2018. Available from: https://doi.org/10.1787/9789264264359-en.

11. Hou F, Xing C, Li B, Cheng J and Chen W: Performance of a novel in vitro assay for skin sensitization based on activation of T lymphocytes ALTEX 37: 451-468, 2020.

12. Croft $\mathrm{M}$ and Dubey C: Accessory molecule and costimulation requirements for CD4 T cell response. Crit Rev Immunol 37: 261-290, 2017.

13. Abbas AK, Trotta E, Simeonov DR, Marson A and Bluestone JA: Revisiting IL-2: Biology and therapeutic prospects. Sci Immunol 3: eaat 1482,2018

14. Claesson MH, Dissing S, Tscherning T and Geisler C: T-cell activation. V. Anti-major histocompatibility complex class I antibody-induced activation and clonal abortion in Jurkat T-leukaemic cells. Immunology 78: 444-448, 1993.

15. Stecha P, Grailer J, Cheng JJ, Hartnett J, Fan F and Stecha MC: American Association of Cancer Research (AACR): Abstract 5439: Development of a robust reporter-based T-cell activation assay for bispecific therapeutic antibodies in immunotherapy. Cancer Ref: Aug 12, 2015 (Epub ahead of print).

16. Takenouchi O, Fukui S, Okamoto K, Kurotani S, Imai N, Fujishiro M, Kyotani D, Kato Y, Kasahara T, Fujita M, et al: Test battery with the human cell line activation test, direct peptide reactivity assay, and DEREK based on a 139 chemical data set for predicting skin sensitizing potential and potency of chemicals. J Appl Toxicol 35: 1318-1332, 2015.

17. Exon JH, KollerLD, TalcottPA, O'Reilly CA and Henningsen GM: Immunotoxicity testing: an economical multiple-assay approach. Fundam Appl Toxicol 7: 387-397, 1986.

18. Rooney AA, Luebke RW, Selgrade MK and Germolec DR: Immunotoxicology and its application in risk assessment. Exp Suppl 101: 251-287, 2012.

19. Esser PR and Martin SF: Pathomechanisms of contact sensitization. Curr Allergy Asthma Rep 17: 83, 2017.

20. Saito R, Hirakawa S, Ohara H, Yasuda M, Yamazaki T, Nishii S and Aiba S: Nickel differentially regulates NFAT and NF- $\kappa B$ activation in T cell signaling. Toxicol Appl Pharmacol 254: 245-255, 2011.

21. Park KR, Lee JH, Choi CY, Liu KH, Seog DH, Kim YH, Kim DE, Yun $\mathrm{CH}$ and Yea SS: Suppression of interleukin-2 gene expression by isoeugenol is mediated through down-regulation of NF-AT and NF-kappaB. Int Immunopharmacol 7: 1251-1258, 2007.

22. Ahn J, Avonto C, Chittiboyina AG and Khan IA: Is isoeugenol a prehapten? Characterization of a Thiol-reactive oxidative byproduct of isoeugenol and potential implications for skin sensitization. Chem Res Toxicol 33: 948-954, 2020.

23. Barratt MD and Basketter DA: Possible origin of the skin sensitization potential of isoeugenol and related compounds. (I) Preliminary studies of potential reaction mechanisms. Contact Dermatitis 27: 98-104, 1992.

24. Clouet E, Bechara R, Raffalli C, Damiens MH, Groux H, Pallardy M, Ferret PJ and Kerdine-Römer S: The THP-1 cell toolbox: A new concept integrating the key events of skin sensitization. Arch Toxicol 93: 941-951, 2019.

25. Organisation for Economic Co-operation and Development: Guideline No. 497D: Defined Approaches on Skin Sensitisation. 2021. Available from: https://www.oecd.org/env/guideline-no-4 97-defined-approaches-on-skin-sensitisation-b92879a4-en.htm.

26. van der Valk J, Bieback K, Buta C, Cochrane B, Dirks WG, Fu J, Hickman JJ, Hohensee C, Kolar R, Liebsch M, et al: Fetal Bovine Serum (FBS): Past-Present-Future. ALTEX 35: 99-118, 2018.

27. Weber $\mathrm{T}$ and Wagner K: Replacing fetal bovine serum (FBS) in research and testing. ALTEX 38: 163-164, 2021.

This work is licensed under a Creative Commons Attribution-NonCommercial-NoDerivatives 4.0 International (CC BY-NC-ND 4.0) License. 